\title{
Influence of camera distortions on satellite image registration and change detection applications
}

\author{
François Ayoub*, Sébastien Leprince*, Renaud Binet ${ }^{\dagger}$, Kevin W. Lewis*, Oded Aharonson*, \\ and Jean-Philippe Avouac* \\ *Division of Geological and Planetary Sciences \\ California Institute of Technology \\ MC100-23, 1200 E. California Blvd, Pasadena, CA 91125, USA \\ Phone: (626) 395-3726, Email: fayoub@gps.caltech.edu \\ ${ }^{\dagger}$ Laboratoire de Détection et Géophysique \\ Commissariat à l'Energie Atomique \\ 91297 Arpajon, France
}

\begin{abstract}
Applications such as change detection and digital elevation model extraction from optical images require a rigorous modeling of the acquisition geometry. We show that the unrecorded satellite jitter during image acquisition, and the uncertainties on the CCD arrays geometry are the current major limiting factors for applications requiring high accuracy. These artifacts are identified and quantified on several optical satellites, i.e., SPOT, ASTER, Quickbird, and HiRise.

Index Terms-Change detection, subpixel correlation, distor-
\end{abstract} tion, jitter, geometric error

\section{INTRODUCTION}

Accurate geometrical modeling of optical imaging systems is of prime importance to finely monitor the Earth's surface. This is particularly relevant to digital elevation model (DEM) generation, measurement of surface displacement (iceflow, landslide, coseismic deformation), and change detection applications. Recent methodological advances now allow for the co-registration of pushbroom satellite images with $1 / 50$ pixel accuracy, which makes possible the measurement of ground displacement with accuracy better than $1 / 10$ of the pixel size [1]. This methodology was implemented in the Co-registration of Optically Sensed Images and Correlation $(\text { COSI-Corr })^{1}$ software package. As a drawback of such measurement accuracy, uncertainties on the imaging system are exacerbated, and unmodeled distortions may severely affect applications requiring high accuracy.

Here, we characterize the unmodeled distortions of several optical sensors currently in use, and we pinpoint the possible consequences for different applications. Multi-temporal images of the same area and from the same sensor are orthorectified, co-registered and correlated with COSI-Corr according to [1]. The procedure applies a rigorous modeling using all available acquisition data (e.g. camera geometry, spacecraft attitudes, orbits). If the image's geometries were correctly modeled, the correlation map should be uniformly zero. However, tests ran on images from the SPOT, ASTER, Quickbird, and HiRISE satellites, revealed clear artifacts originating from

\footnotetext{
${ }^{1}$ URL: http://www.tectonics.caltech.edu/slip_history/spot_coseis/
}

focal plane distortions and from unmodeled platform attitudes (jitter).

\section{SPOT 1,2,3 AND 4}

The SPOT (Satellite Pour l'Observation de la Terre) constellation comprises 5 satellites. The latest satellite, SPOT 5, has its panchromatic optical detector made of an unique CCD array, whereas the SPOT 1, 2, 3, and 4 detectors are composed of $4 \mathrm{CCD}$ arrays combined together through an optical divider. The accurate knowledge of the CCD arrays alignment is essential for a geometrically correct image reconstruction. In [1], the ground deformation produced by the 1999, Mw 7.1, Hector Mine earthquake was retrieved through the correlation of a $10 \mathrm{~m}$ SPOT 2 and a $10 \mathrm{~m}$ SPOT 4 images bracketing the event. The standard deviation of the measurements was around $80 \mathrm{~cm}$, and the coseismic ground deformation could be localized and measured. However, the measurements were polluted with along-track stripes from CCD misalignments whose amplitudes were up to $1.6 \mathrm{~m}$.

The calibration of these CCD misalignments was studied in [2] and applied to the SPOT 4 HRV1 sensor. The ground distortion induced by each CCD array was measured using subpixel correlation between the SPOT 4 HRV1 image and a SPOT 5 image that was assumed distortion-free. The images were precisely orthorectified and coregistered before correlation to account for the images' acquisition geometries and to remove any stereoscopic effect. Distortions found in the disparity map were then reprojected in the camera focal plane. CCD arrays misalignments were reduced by one order of magnitude (Fig. 1). As of now, the refinement of the SPOT 4 HRV1 and SPOT 2 HRV1 CCD arrays orientation were processed and implemented in the COSI-Corr package.

\section{ASTER}

ASTER (Advanced Spaceborne Thermal Emission and Reflection Radiometer) is an imaging instrument flying on Terra, a satellite launched in December 1999 as part of NASA's Earth Observing System (EOS). The ASTER Visible Near Infrared (VNIR) device is composed of an unique CCD array 


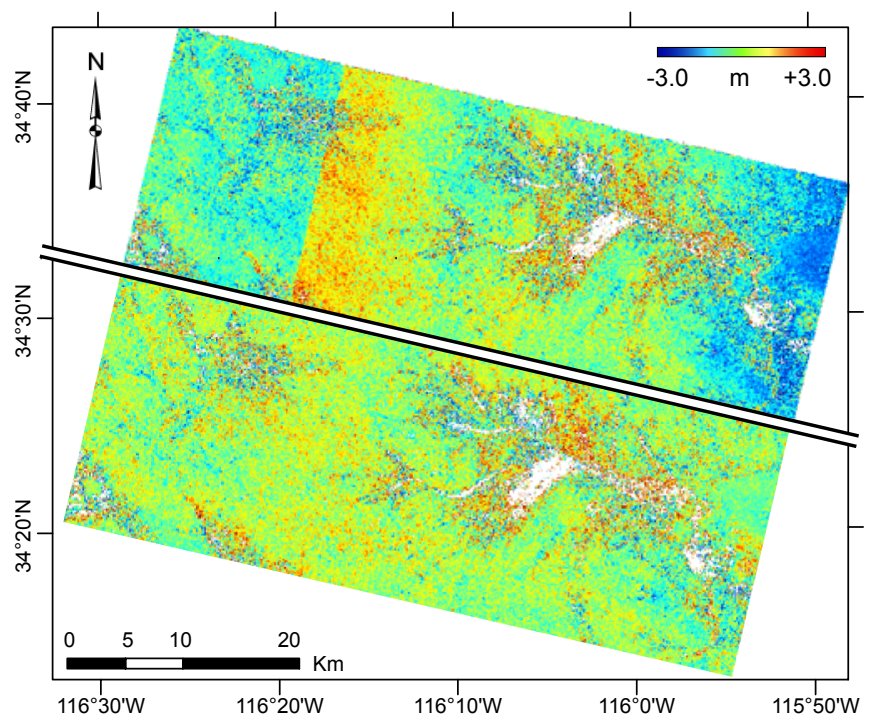

Fig. 1: East-West component (eastward positive) of two disparity fields measured from subpixel correlation of a reference 5$m$ SPOT 5 image and a 10-m SPOT 4 image near Hector Mine (California). Top: the camera geometry provided by SPOT Image Corp. was accounted for. Linear artifacts from SPOT 4 CCD misalignments are identifiable with amplitude greater than $1 \mathrm{~m}$. Bottom: the disparity field was generated using the same images but the SPOT 4 CCD arrays misalignments were calibrated according to [2]. Correlation analysis were performed on $32 \times 32$ pixel windows, sliding with a step of 8 pixels.

for each spectral band. In a context of tectonic investigation, an ASTER image acquired on May 10, 2000 was correlated with a SPOT 4 image acquired August 17, 1998, with the purpose of measuring the coseismic ground deformation sustained by the 1999, Mw 7.1, Hector Mine earthquake. Prior to correlation, the images were orthorectified and co-registered on a $10 \mathrm{~m}$ UTM grid using the methodology described in [1]. The SPOT 4 CCD arrays alignments were refined as explained in II.

On the East/West displacement map (Fig. 2), the fault discontinuity is readily identifiable. However, a strong, undulating pattern is corrupting the displacement map and impairs quantitative measurement of the coseismic displacement. This pattern has an amplitude of around 6-7 $\mathrm{m}$ and a frequency of $1.6 \mathrm{~Hz}$. This artifact is attributed to the ASTER image only. Indeed, in [1], the same SPOT image was correlated with a non ASTER image, and no similar pattern was detected. This artifact is a consequence of unrecorded spacecraft jitter during the ASTER image acquisition.

The Terra on-board attitude recorders have a sensitivity of 1 arcsecond (around $3.4 \mathrm{~m}$ on ground for VNIR images) and a sampling rate of $0.97 \mathrm{~Hz}$. The sampling rate is insufficient to record properly the spacecraft jitter observed on Fig. 2. The displacement generated from the jitter has an amplitude greater than the devices sensitivity, and should have been

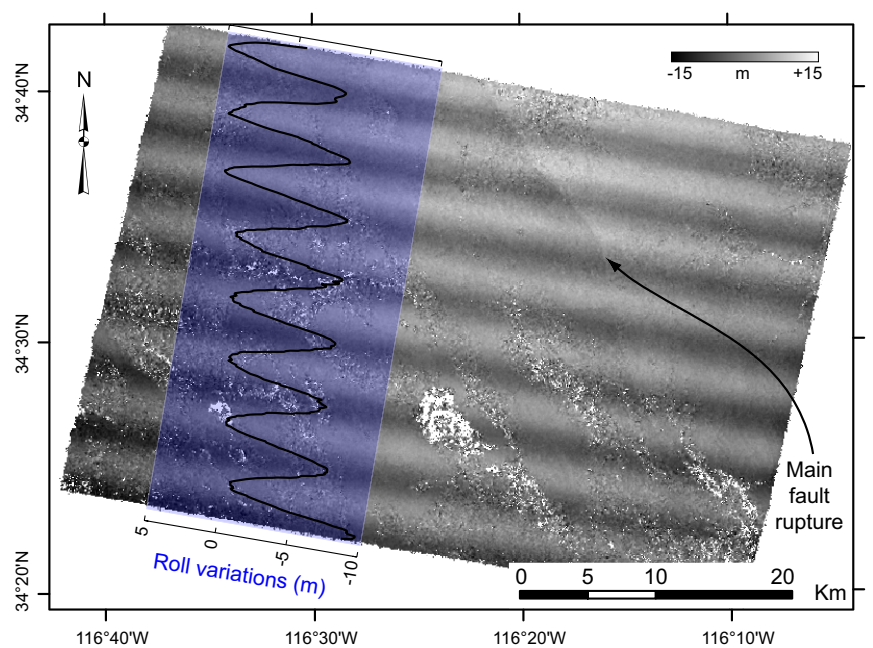

Fig. 2: Disparity map in the East/West direction between a SPOT 4 and an ASTER images, acquired on May 10, 2000, and on August 17, 1998 over the Hector Mine area, California. Images were orthorectified on a $10 \mathrm{~m}$ resolution UTM projection, and subpixel correlation was performed on $32 \times 32$ pixels windows, sliding with a step of 8 pixels. The SPOT 4 image was corrected for CCD arrays misalignments as explained in II. The goal of this study was to detect and measure the ground rupture produced during the 1999, Mw 7.1, Hector Mine earthquake. The fault rupture is identifiable but accurate measurements are difficult due to the oscillating bias induced by the unmodeled jitter of the ASTER spacecraft during the image acquisition.

detected. The devices are either malfunctioning, or, possibly, the undersampled measurements provide an aliased jitter record. Similar artifacts in amplitude and frequency were also reported in [3], [4], and [5]. The latter shows in addition that DEMs extracted from ASTER imagery are distorted with such artifacts.

\section{QUICKBIRD}

Launched in 2001, the Quickbird satellite provides images with the highest resolution available to the general public. Its panchromatic detector is composed of six CCD arrays, alternatively placed aft and forth in the focal plane, and acquires images with a ground sampling of $60 \mathrm{~cm}$. In [6], two SPOT 5 - $2.5 \mathrm{~m}$ resolution images with similar viewing angles were correlated to reveal the location and to provide estimates of the surface offsets induced by the December 26, 2003, Mw 6.6 Bam earthquake in Iran. Building on this study, we applied a similar technique using Quickbird panchromatic imagery to refine the SPOT derived measurements. Unfortunately, the large viewing angle difference between the Quickbird images $\left(14.3^{\circ}\right)$ produced significant topography residuals in the correlation maps, that could not be corrected for using the $3-\operatorname{arcsec}(90 \mathrm{~m})$ SRTM DEM. We therefore resampled and co-registered both Quickbird images at $50 \mathrm{~cm}$ resolution in an epipolar geometry. Cross-correlation in this geometry leads 
to a displacement map with a component that supposedly only depends on the topography, while the other component is independent of the topography. This is this last component that we present in Fig. 3. We show that the fault rupture can indeed be recovered but that, unfortunately, residual distortions from the imaging system corrupt the measurements. A CCD discontinuity artifacts is indeed aligned with the fault rupture, mixing both signals. Unmodeled jitter produces distortion of around 5 pixels $(2.5 \mathrm{~m})$ which occurs mainly around $1 \mathrm{~Hz}$. We also notice a jitter of $4.3 \mathrm{~Hz}$ of amplitude 0.2 pixel. Finally, we notice seam artifacts between CCD arrays that are produced during the image stitching process. Indeed, adjacent CCD arrays do slightly overlap and the necessary merging of overlapping areas involves a registering step subject to errors.

\section{HIRISE}

Since 2006, the High Resolution Imaging Science Experiment (HiRise) on board of the Mars Reconnaissance Orbiter (MRO) images the Mars surface with unprecedented resolution (around $30 \mathrm{~cm}$ ) [7]. The monochromatic (red wavelength) optical detector is composed of $10 \mathrm{CCD}$ arrays, alternatively placed aft and forth in the focal plane, and are slightly overlapping to insure a continuous ground imaging between CCD arrays. Two multi-temporal overlapping images, acquired over the Victoria crater in the Meridiani Planum, were retrieved in raw format (EDR) from the HiRise website and reconstructed in the focal plane geometry. The reconstruction accounted for the acquisition parameters: radiometry, camera geometry, spacecraft attitudes and ephemerides (SPICE kernels). Topography was not accounted for, but the identical viewing angles between the two images removes stereoscopic effects. The reconstructed images were then co-registered and correlated.

The along-track disparity map (Fig. 4) shows strong geometric distortions in column and line directions. The undulating pattern (red) reveals spacecraft jitter that was unmodeled during the images reconstruction. The vertical stripes (blue) originate, potentially, from two causes. The first cause would be the consequence of the unmodeled jitter on the staggered CCD arrays. Indeed, unmodeled jitter introduces a relative shift between images acquired by adjacent, staggered, CCD arrays. The amplitude of these shift depends on both the jitter characteristics (frequency and amplitude), and the time delay between adjacent CCD arrays to image a same ground location. The second cause would be a CCD arrays misalignment with respect to their nominal position. In the latter case, the absolute CCD array orientation in in the focal plane proposed by [2] is limited here as no high resolution reference is available. However, a relative orientation between CCD arrays could be operated.

The amplitude of the jitter distortion is up to 3 pixels (around $1 \mathrm{~m}$ ), whereas the amplitude of the CCD arrays induced distortion is around 4-5 pixels (1-1.5 m). The cross-track disparities map, not displayed here, presents the same artifacts with about the same amplitude. These artifacts can corrupt applications requiring precise photogrammetry. Indeed, we produced a DEM from HiRise imagery using ISIS3 (USGS)

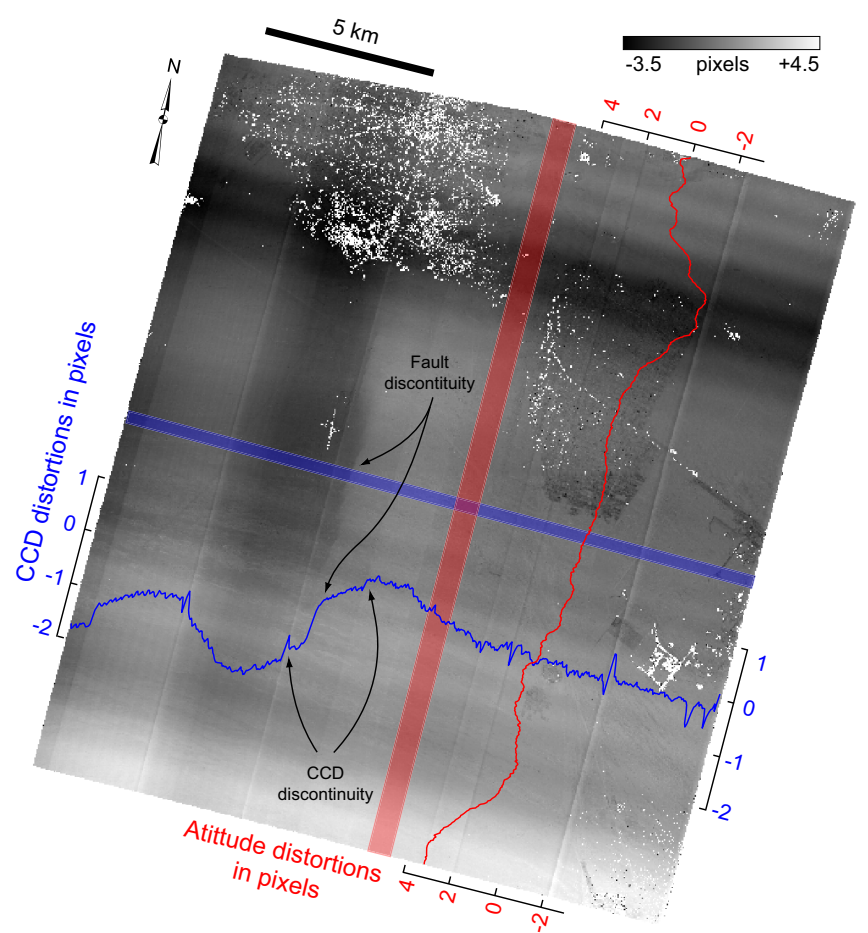

Fig. 3: Disparity map in the direction orthogonal to the epipolar direction between two Quicbird images (03SEP30063637P1BS-000000170456_01_P001 and 04JAN03064311-P1BS000000170455_01_P001), acquired above the city of Bam, Iran, on September 30, 2003, and on January 03, 2004. Images were resampled at a $50 \mathrm{~cm}$ resolution and subpixel correlation was performed on $128 \times 128$ pixels windows, sliding with a step of 64 pixels. This disparity map should not show any particular pattern, and indeed, no topography signal is detected. The goal of this study was to detect and to measure the ground rupture produced during the $\mathrm{Mw} 6.6$ Bam earthquake of December 26, 2003. In this geometry, the fault rupture is measured to be less than 2 pixels (less than $1 \mathrm{~m}$ ), but accurate measurements are difficult due to the bias imposed by distortions from the imaging system such as CCD interconnection discontinuity and jitter artifacts. In particular, a CCD distortion seem to exactly coincide with the coseismic signal to be measured. White areas are decorrelation areas, mostly produced by the collapse of buildings during the earthquake.

and SOCET SET (BAE Systems) softwares and applying the procedure defined by the HiRise team. CCD arrays artifacts, similar to the ones found in Fig. 4, were identified on the DEM as seen on Fig. 5, and generate an elevation error of around $1 \mathrm{~m}$. In [8], DEM extraction with HiRise imagery was confronted to similar CCD arrays and jitter artifact.

\section{CONCLUSION}

This study reveals that geometrical distortions of current pushbroom satellites are to be sought among two main causes: the modeling inaccuracy of the CCD sensor geometry, and 


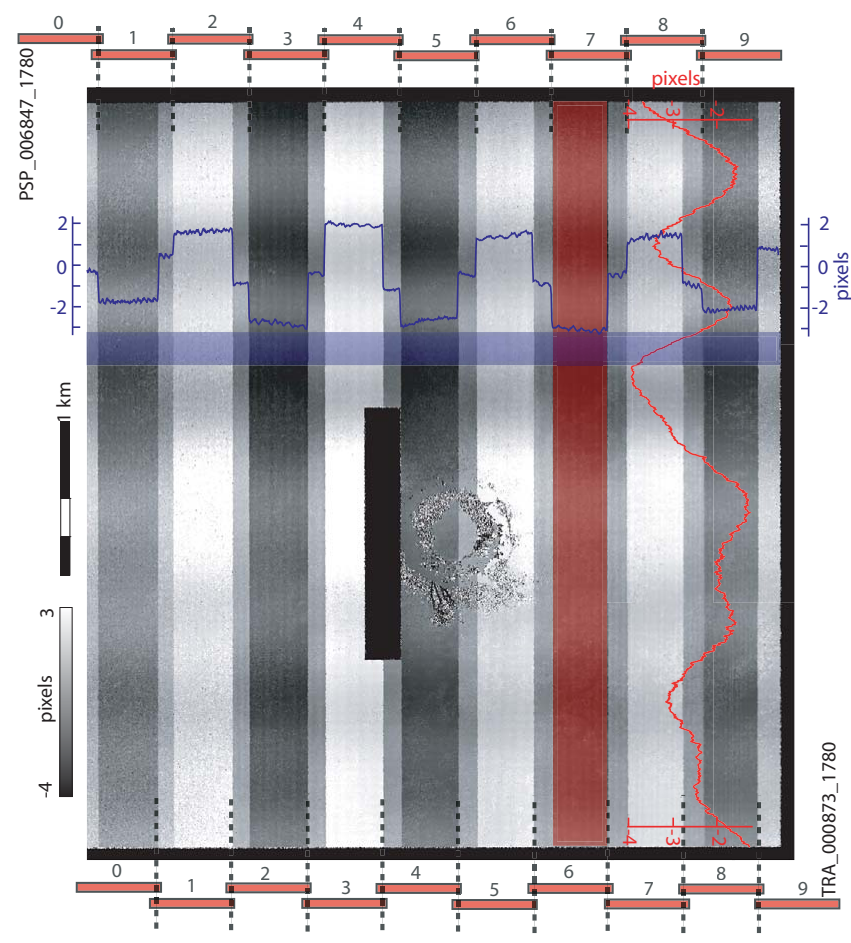

Fig. 4: Along-track component (up positive) of the disparity field measured from subpixel correlation of two $25-\mathrm{cm}$ HiRISE images (TRA_000873_1780 and PSP_006847_1780), acquired over the Victoria crater area in Meridiani Planum. The reconstruction accounted for the images radiometry, camera geometry, spacecraft attitudes and ephemerides. Topography was not accounted for, but the identical incidence angles removes any stereoscopic effect. They were correlated using a window size of $64 \times 64$ pixels and a sliding step of 16 pixels. The black stripe is due to missing data in the PSP_006847_1780 image, and the decorrelation area right of the black stripe is caused by large areas covered with fine material that appear featureless at the correlation window size. As expected, no topographic effect are noticed, but strong artifacts are found in the column and line directions. Crosstrack artifacts (red profile averaged in line direction over the red box) reveal unmodeled spacecraft jitter, while along-track artifacts (blue profile averaged in column direction over the blue box) are produced by a probable combination of CCD arrays misalignment and unmodeled jitter on staggered CCD arrays. The CCD arrays' relative position of the two images are sketched above and below the disparity field.

the jitter of the instrument's platform during image acquisition. These two problems occur on most pushbroom systems, whether they orbit the Earth or Mars, and whether they deliver low- or high-resolution images. Artifacts induced in disparity maps hamper change detection applications as well as DEM generation. Proper correction and modeling of these distorting causes is becoming an urgent need since these distortions may now be greater than the images resolution, limiting the potential of high-resolution systems. Solutions to correct for

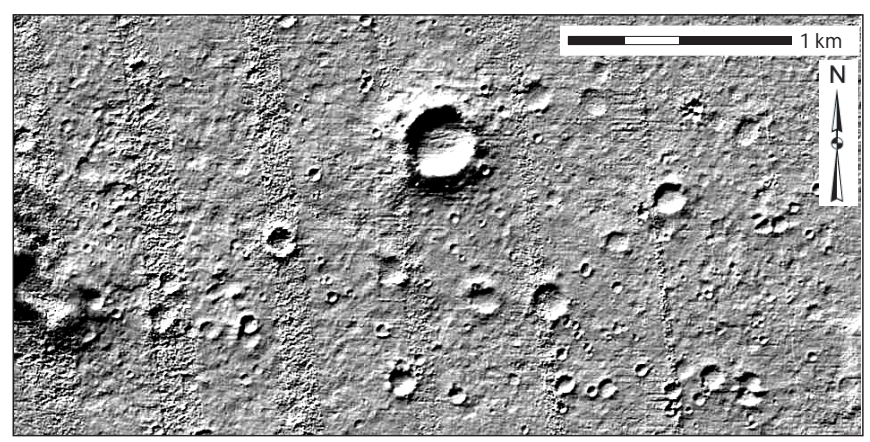

Fig. 5: Shaded DEM generated from HiRISE imagery near Columbia Hills. Raw images were reconstructed using the USGS ISIS-3 software and the corresponding SPICE kernels, and DEM generation was operated using the SOCET-SET software. Although the camera geometry was recently refined, residual CCD misalignments generate artifacts in the DEM with an amplitude of around $1 \mathrm{~m}$.

these distortions are appearing [2], [9], [10], and precisely detecting and characterizing them is indeed a decisive step to this end.

\section{REFERENCES}

[1] S. Leprince, S. Barbot, F. Ayoub, and J. P. Avouac, "Automatic and precise ortho-rectification, co-registration and sub-pixel correlation of satellite images, application to ground deformation measurements," IEEE Trans. Geosci. Remote Sens., vol. 45, pp. 1529-1558, 2007.

[2] S. Leprince, P. Musé, and J. P. Avouac, "In-flight ccd distortion calibration for pushbroom satellites based on subpixel correlation," 2007, submitted.

[3] J. P. Avouac, F. Ayoub, S. Leprince, O. Konca, and D. Helmberger, "The 2005, mw 7.6 kashmir earthquake, rupture kinematics from subpixel correlation of aster images and seismic waveforms analysis," Earth Planet. Sci. Lett., vol. 249, pp. 514-528, 2006.

[4] S. Leprince, F. Ayoub, Y. Klinger, and J. P. Avouac, "Co-registration of optically sensed images and correlation (cosi-corr): an operational methodology for ground deformation measurements," in Int. Geosci. Remote Sens. Symposium (IGARSS), vol. 6, Barcelona, Spain, July 2007, pp. 2700-2702.

[5] A. Iwasaki and H. Fujisada, "Image correlation tool for aster geometric validation," in Proc. SPIE, vol. 4881, 2002, pp. 111-120.

[6] R. Binet and L. Bollinger, "Horizontal coseismic deformation of the 2003 bam (iran) earthquake measured from spot-5 thr satellite imagery," Geophys. Res. Lett., 2005, 10.1029/2004GL021897.

[7] A. S. McEwen, E. M. Eliason, J. W. Bergstrom, N. T. Bridges, C. J. Hansen, W. A. Delamere, J. A. Grant, V. C. Gulick, K. E. Herkenhoff, L. Keszthelyi, R. L. Kirk, M. T. Mellon, S. W. Squyres, N. Thomas, and C. M. Weitz, "Mars reconnaissance orbiter's high resolution imaging science experiment (hirise)," J. Geophys. Res., 2007 doi:10.1029/2005JE002605.

[8] R. L. Kirk, E. Howington-Kraus, M. R. Rosiek, D. Cook, J. Anderson, K. Becker, B. A. Archinal, L. Keszthelyi, R. King, A. S. McEwen, and H. Team, "Ultrahigh resolution topographic mapping of mars with hirise stereo images: Methods and first results," in Seventh International Conference on Mars, July 9-13 2007, p. 3381, 1PI Contribution No. 1353.

[9] Y. Teshima and A. Iwasaki, "Correction of attitude fluctuation of terra spacecraft using aster/swir imagery with parallax observation," IEEE Trans. Geosci. Remote Sens., vol. 46, pp. 222-227, 2008.

[10] D. C. Bamber, P. Palmer, and S. Mackin, "High performance attitude determination through analysis of geometric distortions within earth observational satellite imagery," in 20th Annual AIAA/USU Conference on small satellites, Utah, 2006. 